PROCEEDINGS OF THE

AMERICAN MATHEMATICAL SOCIETY

Volume 130, Number 3, Pages 697-702

S 0002-9939(01)06123-8

Article electronically published on June 20, 2001

\title{
ON NORMAL OPERATOR EXPONENTIALS
}

\author{
CHRISTOPH SCHMOEGER
}

(Communicated by Joseph A. Ball)

\begin{abstract}
Suppose that $A$ and $B$ are bounded normal operators on a complex Hilbert space and that $e^{A} e^{B}=e^{B} e^{A}$. In this paper some conditions implying $A B=B A$ are given.
\end{abstract}

\section{TERminology AND RESUlts}

Throughout this paper let $\mathcal{H}$ denote a complex Hilbert space and $\mathcal{L}(\mathcal{H})$ the Banach algebra of all bounded linear operators on $\mathcal{H}$. For $A \in \mathcal{L}(\mathcal{H})$ the spectrum and the spectral radius of $A$ are denoted by $\sigma(A)$ and $r(A)$, respectively. The set of eigenvalues of $A$ is denoted by $\sigma_{p}(A)$. For the resolvent set of $A$ we write $\rho(A)$. We use $N(A)$ and $A(\mathcal{H})$ to denote the kernel and the range of $A$, respectively.

We say that $\sigma(A)$ is $2 \pi i$-congruence-free if

$$
\sigma(A) \cap \sigma(A+2 j \pi i)=\emptyset \text { for } j= \pm 1, \pm 2, \ldots .
$$

If $A \in \mathcal{L}(\mathcal{H})$ is normal and has the spectral resolution

$$
A=\int_{\sigma(A)} \lambda d E(\lambda),
$$

let $E(\Omega)$ denote the associated projection measure defined on the Borel subsets $\Omega \subseteq \sigma(A)$. It is convenient to think of $E$ as being defined for all Borel sets in $\mathbb{C}$ : put $E(\Omega)=E(\Omega \cap \sigma(A))$.

Definition. Let $A \in \mathcal{L}(\mathcal{H})$ be normal with the spectral resolution (1.1). We say that $\sigma(A)$ is generalized $2 \pi i$-congruence-free if

$$
E(\sigma(A) \cap \sigma(A+2 j \pi i))=0 \text { for } j=1,2, \ldots .
$$

Remarks. Let $A \in \mathcal{L}(\mathcal{H})$ be normal with the spectral resolution (1.1).

(1) Since $E(\emptyset)=0$, it is clear that if $\sigma(A)$ is $2 \pi i$-congruence-free, then $\sigma(A)$ is generalized $2 \pi i$-congruence-free.

(2) Let $n$ denote the smallest integer with $n>\frac{r(A)}{\pi}$. Then $\sigma(A) \cap \sigma(A+2 j \pi i)=\emptyset$ for $j \geq n$. Thus (1.2) holds if and only if

$$
E(\sigma(A) \cap \sigma(A+2 j \pi i))=0 \text { for } j=1,2, \ldots, n-1 .
$$

Received by the editors April 3, 2000 and, in revised form, August 20, 2000.

2000 Mathematics Subject Classification. Primary 47A10, 47A60.

Key words and phrases. Normal operators, exponentials. 
Examples. Let $A \in \mathcal{L}(\mathcal{H})$ be normal with the spectral resolution (1.1).

(1) If $r(A)<\pi$, then (1.2) holds.

(2) Let $\pi \leq r(A)<2 \pi$. By Remark (2), (1.2) holds if and only if

$$
E(\sigma(A) \cap \sigma(A+2 \pi i))=0 .
$$

(3) Let $2 \pi \leq r(A)<3 \pi$. By Remark (2), (1.2) holds if and only if

$$
E(\sigma(A) \cap \sigma(A+2 \pi i))=E(\sigma(A) \cap \sigma(A+4 \pi i))=0 .
$$

(4) Let $j \in \mathbb{N}$ and suppose that there is some $\mu \in \mathbb{C}$ with $\sigma(A) \cap \sigma(A+2 j \pi i) \subseteq\{\mu\}$ and $\mu \notin \sigma_{p}(A)$. Since $E(\{\mu\})=0$ (44, Theorem 12.29]), it follows that

$$
E(\sigma(A) \cap \sigma(A+2 j \pi i))=0 .
$$

The following result is due to E. M. E. Wermuth ([8]). See [6] for a very short proof.

Theorem 1.1. Let $A, B \in \mathcal{L}(\mathcal{H})$ and suppose that $\sigma(A), \sigma(B)$ are $2 \pi i$-congruencefree. If $e^{A} e^{B}=e^{B} e^{A}$, then $A B=B A$.

If $\sigma(A)$ or $\sigma(B)$ is not $2 \pi i$-congruence-free, then, in general, $e^{A} e^{B}=e^{B} e^{A}$ does not imply that $A B=B A$. For examples see [6], [7] and [8].

But for normal operators we can say more. Now we state the main results of this paper. Proofs will be given in Section 3 of this paper.

Theorem 1.2. Suppose that $A$ and $B$ are normal operators in $\mathcal{L}(\mathcal{H})$ and suppose that $e^{A} e^{B}=e^{B} e^{A}$.

(a) If $\sigma(A)$ is generalized $2 \pi i$-congruence-free, then $A e^{B}=e^{B} A$.

(b) If $\sigma(A)$ and $\sigma(B)$ are generalized $2 \pi i$-congruence-free, then $A B=B A$.

Theorem 1.3. Let $A, B \in \mathcal{L}(\mathcal{H})$. If $A+B$ is normal, if $\sigma(A+B)$ is generalized $2 \pi i$-congruence-free and if

$$
e^{A} e^{B}=e^{A+B}=e^{B} e^{A},
$$

then $A B=B A$.

We introduce the following notation: for $B \in \mathcal{L}(\mathcal{H})$ write $\{B\}^{c}$ for the set

$$
\{T \in \mathcal{L}(\mathcal{H}): T B=B T\} .
$$

The set $\{B\}^{c c}$ is defined by

$$
\{B\}^{c c}=\left\{S \in \mathcal{L}(\mathcal{H}): S T=T S \text { for all } T \in\{B\}^{c}\right\} .
$$

Theorem 1.4. Let $A \in \mathcal{L}(\mathcal{H})$ be normal and suppose that $\sigma(A)$ is generalized $2 \pi i$ congruence-free. If $B \in \mathcal{L}(\mathcal{H})$ and $e^{A}=e^{B}$, then $A \in\{B\}^{c c}$ (and so $A B=B A$ ).

Theorem 1.5. Let $A, B \in \mathcal{L}(\mathcal{H})$. If $A$ is selfadjoint, $\sigma(A) \subseteq[-\pi, \pi]$ and $e^{i A}=e^{B}$, then we have

(a) $B^{*}=-B$ if $B$ is normal,

(b) $A \in\{B\}^{c c}$ (and so $A B=B A$ ), if $-\pi \notin \sigma_{p}(A)$ or $\pi \notin \sigma_{p}(A)$.

Observe that part (b) of Theorem 1.5 is a generalization of [5, Satz 4 (b)]. 


\section{Preparations}

In order to prove the theorems in Section 1 we need some supplementary results. Let $A \in \mathcal{L}(\mathcal{H})$. The $\operatorname{map} \delta_{A}: \mathcal{L}(\mathcal{H}) \rightarrow \mathcal{L}(\mathcal{H})$, defined by

$$
\delta_{A}(C)=C A-A C(C \in \mathcal{L}(\mathcal{H})),
$$

is called the inner derivation determined by $A$. It is clear that $\delta_{A}$ is a bounded linear operator on $\mathcal{L}(\mathcal{H})$ with $\left\|\delta_{A}\right\| \leq 2\|A\|$. It is shown in [1] that

$$
\sigma\left(\delta_{A}\right)=\{\lambda-\mu: \lambda, \mu \in \sigma(A)\} .
$$

From [2] Proposition 6.4.8] it follows that

$$
e^{\delta_{A}}(C)=e^{-A} C e^{A} \text { for all } C \in \mathcal{L}(\mathcal{H}) .
$$

Throughout this paper let $f$ denote the entire function $f: \mathbb{C} \rightarrow \mathbb{C}$ given by

$$
f(z)= \begin{cases}z^{-1}\left(e^{z}-1\right), & \text { if } z \neq 0 \\ 1, & \text { if } z=0\end{cases}
$$

From $z f(z)=f(z) z=e^{z}-1$ and (2.2) we get

$$
f\left(\delta_{A}\right)\left(\delta_{A}(C)\right)=e^{-A} C e^{A}-C \text { for all } C \in \mathcal{L}(\mathcal{H}) .
$$

Proposition 2.1. Let $A \in \mathcal{L}(\mathcal{H})$, let $n$ denote the smallest integer with $n>\frac{r(A)}{\pi}$ and let

$$
M_{A}=\left\{\lambda \in \sigma\left(\delta_{A}\right): f(\lambda)=0\right\} .
$$

(a) If $\sigma(A)$ is $2 \pi i$-congruence-free, then $M_{A}=\emptyset$.

(b) If $M_{A}=\emptyset$, then $f\left(\delta_{A}\right)$ is an invertible operator on $\mathcal{L}(\mathcal{H})$.

(c) If $\lambda \in M_{A}$, then $\lambda$ is a simple zero of $f$ and there is $j \in \mathbb{Z} \backslash\{0\}$ with $\lambda=2 j \pi i$.

(d) $M_{A}$ has at most $2(n-1)$ elements,

$$
M_{A} \subseteq\{ \pm 2 \pi i, \pm 4 \pi i, \ldots, \pm 2(n-1) \pi i\} .
$$
then

(e) If $M_{A} \neq \emptyset$ and $M_{A}=\left\{\lambda_{1}, \ldots, \lambda_{k}\right\}$ with $k \leq 2(n-1)$ and $\lambda_{j} \neq \lambda_{l}$ for $j \neq l$,

$$
N\left(f\left(\delta_{A}\right)\right)=N\left(\delta_{A}-\lambda_{1}\right) \oplus \cdots \oplus N\left(\delta_{A}-\lambda_{k}\right)
$$

and $C^{n}=0$ for each $C \in N\left(\delta_{A}-\lambda_{j}\right)$.

Proof. (a) clear. (b) is valid, since $f(\lambda) \neq 0$ for all $\lambda \in \sigma\left(\delta_{A}\right)$. (c), (d) and (e) are shown in 7 .

Notation. Let $T \in \mathcal{L}(\mathcal{H})$ and $\Omega \subseteq \mathbb{C}, \Omega \neq \emptyset$. Let $S(T ; \Omega)$ be the subset of $\mathcal{H}$ defined by

$$
S(T ; \Omega)=\bigcap_{\lambda \in \Omega}(T-\lambda)(\mathcal{H}) .
$$

The following propositions are of central importance for our investigation.

Proposition 2.2. If $A \in \mathcal{L}(\mathcal{H})$ is normal and has the spectral resolution (1.1) and if $B \in \mathcal{L}(\mathcal{H})$, then

$$
S(A ; \rho(B))=E(\sigma(A) \cap \sigma(B))(\mathcal{H}) .
$$

Proof. Theorem 1 in [3]. 
Proposition 2.3. Let $A \in \mathcal{L}(\mathcal{H})$ be normal and let $\mu \in \mathbb{C}$. Then

$$
(A-\mu)(\mathcal{H})=\left(A^{*}-\bar{\mu}\right)(\mathcal{H}) .
$$

Proof. Since $A$ is normal, $A-\mu$ is normal. Exercise 12.36 in [4] shows that (2.4) holds.

Proposition 2.4. Let $A \in \mathcal{L}(\mathcal{H})$ be normal and suppose that $A$ has the spectral resolution (1.1).

(a) If $\lambda_{0} \in \mathbb{C}, C \in N\left(\delta_{A}-\lambda_{0}\right)$ and $D \in N\left(\delta_{A}+\lambda_{0}\right)$, then

$$
C(\mathcal{H}) \subseteq E\left(\sigma(A) \cap \sigma\left(A-\lambda_{0}\right)\right)(\mathcal{H})
$$

and

$$
D^{*}(\mathcal{H}) \subseteq E\left(\sigma(A) \cap \sigma\left(A-\lambda_{0}\right)\right)(\mathcal{H}) .
$$

(b) If $\lambda_{0} \in \mathbb{C}$ and $E\left(\sigma(A) \cap \sigma\left(A-\lambda_{0}\right)\right)=0$, then $\lambda_{0}$ and $-\lambda_{0}$ are not eigenvalues of $\delta_{A}$.

(c) If $\sigma(A)$ is generalized $2 \pi i$-congruence-free, then $N\left(f\left(\delta_{A}\right)\right)=\{0\}$.

Proof. (a) Take $C \in \mathcal{L}(\mathcal{H})$ with $C A-A C=\lambda_{0} C$, thus $A C=C\left(A-\lambda_{0}\right)$. Put $B=A-\lambda_{0}$. For $\mu \in \rho(B)$ we get

$$
\begin{aligned}
(A-\mu) C(B-\mu)^{-1} & =A C(B-\mu)^{-1}-\mu C(B-\mu)^{-1} \\
& =C B(B-\mu)^{-1}-\mu C(B-\mu)^{-1} \\
& =C(B-\mu)(B-\mu)^{-1}=C .
\end{aligned}
$$

This shows that $C(\mathcal{H}) \subseteq S(A, \rho(B))$. From Proposition 2.2 we get

$$
S(A, \rho(B))=E(\sigma(A) \cap \sigma(B))(\mathcal{H}),
$$

thus $C(\mathcal{H}) \subseteq E\left(\sigma(A) \cap \sigma\left(A-\lambda_{0}\right)\right)(\mathcal{H})$.

Now take $D \in N\left(\delta_{A}+\lambda_{0}\right)$, hence $D A-A D=-\lambda_{0} D$ thus $D A=\left(A-\lambda_{0}\right) D$. As above let $B=A-\lambda_{0}$. Then $A^{*} D^{*}=D^{*} B^{*}$. A similar computation as above gives

$$
\left(A^{*}-\mu\right) D^{*}\left(B^{*}-\mu\right)^{-1}=D^{*} \text { for all } \mu \in \rho\left(B^{*}\right) \text {. }
$$

Thus $D^{*}(\mathcal{H}) \subseteq S\left(A^{*}, \rho\left(B^{*}\right)\right)$. Since $\rho\left(B^{*}\right)=\{\mu \in \mathbb{C}: \bar{\mu} \in \rho(B)\}$, we get from (2.4) that

$$
S\left(A^{*}, \rho\left(B^{*}\right)\right)=S(A, \rho(B)),
$$

hence, by (2.5), $D^{*}(\mathcal{H}) \subseteq E\left(\sigma(A) \cap \sigma\left(A-\lambda_{0}\right)\right)(\mathcal{H})$.

(b) follows from (a).

(c) Let $n$ be the smallest integer with $n>\frac{r(A)}{\pi}$. Take $\lambda_{0} \in M_{A}$. Thus $\lambda_{0}=2 j \pi i$ with $j \in \mathbb{Z} \backslash\{0\}$ and $|j| \leq n-1$.

Case 1. $j>0$. Since $\sigma(A)$ is generalized $2 \pi i$-congruence-free,

$$
E\left(\sigma(A) \cap \sigma\left(A+\lambda_{0}\right)\right)=0 .
$$

From (b) we then derive $N\left(\delta_{A}-\lambda_{0}\right)=\{0\}$.

Case 2. $j<0$. Then we have

$$
E\left(\sigma(A) \cap \sigma\left(A-\lambda_{0}\right)\right)=0 .
$$

Again use (b) to see that $N\left(\delta_{A}-\lambda_{0}\right)=\{0\}$.

Since $\lambda_{0} \in M_{A}$ was arbitrary, we conclude from Proposition 2.1 (e) that $N\left(f\left(\delta_{A}\right)\right)$ $=\{0\}$. 


\section{Proofs}

Proof of Theorem 1.2. (a) From (2.3) we get

$$
f\left(\delta_{A}\right)\left(\delta_{A}\left(e^{B}\right)\right)=e^{-A} e^{B} e^{A}-e^{B}=0,
$$

thus $\delta_{A}\left(e^{B}\right)=e^{B} A-A e^{B} \in N\left(f\left(\delta_{A}\right)\right)$. Proposition 2.4 (c) gives $A e^{B}=e^{B} A$.

(b) From (a) and (2.3) we get

$$
f\left(\delta_{B}\right)\left(\delta_{B}(A)\right)=e^{-B} A e^{B}-A=0,
$$

therefore, $A B-B A \in N\left(f\left(\delta_{B}\right)\right)$. Hence, by Proposition 2.4 (c), $A B=B A$.

Proof of Theorem 1.3. Use (2.3) to derive

$$
\begin{aligned}
f\left(\delta_{A+B}\right)\left(\delta_{A+B}\left(e^{A}\right)\right) & =e^{-(A+B)} e^{A} e^{A+B}-e^{A} \\
& =e^{-B} e^{-A} e^{A} e^{B} e^{A}-e^{A} \\
& =0 .
\end{aligned}
$$

Proposition 2.4 (c) implies that $e^{A}(A+B)=(A+B) e^{A}$, therefore, $B e^{A}=e^{A} B$. Using (2.3), this gives

$$
\begin{aligned}
f\left(\delta_{A+B}\right)\left(\delta_{A+B}(B)\right) & =e^{-(A+B)} B e^{A+B}-B \\
& =e^{-B} e^{-A} B e^{A} e^{B}-B \\
& =0 .
\end{aligned}
$$

Since $N\left(f\left(\delta_{A+B}\right)\right)=\{0\},(A+B) B=B(A+B)$. This shows that $A B=B A$.

Proof of Theorem 1.4. Let $T \in\{B\}^{c}$. (2.3) implies that

$$
f\left(\delta_{A}\right)\left(\delta_{A}(T)\right)=e^{-A} T e^{A}-T=e^{-B} T e^{B}-T=0 .
$$

Since $N\left(f\left(\delta_{A}\right)\right)=\{0\}$ (Proposition $2.4(\mathrm{c})$ ), we have $A T=T A$. Since $T \in\{B\}^{c}$ was arbitrary, it follows that $A \in\{B\}^{c c}$.

Proof of Theorem 1.5. (a) From $e^{i A}=e^{B}$ we get $e^{-i A}=\left(e^{i A}\right)^{-1}=\left(e^{B}\right)^{-1}=e^{-B}$ and $e^{-i A}=\left(e^{i A}\right)^{*}=\left(e^{B}\right)^{*}=e^{B^{*}}$, hence $e^{B^{*}}=e^{-B}$, thus $e^{B+B^{*}}=I=e^{0}$, since $B$ is normal. Since $B+B^{*}$ is selfadjoint, Corollary 2 in 7 ] gives $B+B^{*}=0$.

(b) Observe that $i A$ is normal and $r(i A) \leq \pi$. It is easy to see that

$$
\sigma(i A) \cap \sigma(i A+2 \pi i) \subseteq\{i \pi\} .
$$

Case 1. $\pi \notin \sigma_{p}(A)$. Then $i \pi \notin \sigma_{p}(i A)$. Example (4) in Section 1 gives

$$
E(\sigma(i A) \cap \sigma(i A+2 \pi i))=0 .
$$

Example (2) in Section 1 shows that $\sigma(i A)$ is generalized $2 \pi i$-congruence-free. Now use Theorem 1.4 to get $A \in\{B\}^{c c}$.

Case 2. $-\pi \notin \sigma_{p}(A)$. Then $\pi \notin \sigma_{p}(-A)$. Since $e^{-i A}=e^{-B}$, we get $A \in\{B\}^{c c}$ as in Case 1.

\section{REFERENCES}

[1] G. Lumer and M. Rosenblum: Linear operator equations. Proc. Amer. Math. Soc. 10 (1959), 32-41. MR 21:2927

[2] T. W. Palmer: Banach algebras and the general theory of ${ }^{*}$-algebras. Vol. I, Cambridge University Press, 1994. MR 95c:46002

[3] C. R. Putnam: Ranges of normal and subnormal operators. Michigan Math. J. 18 (1971), 33-36. MR 43:2550

[4] W. Rudin: Functional Analysis. Second edition, McGraw-Hill (1991). MR 92k:46001 
[5] Ch. Schmoeger: Über die Eindeutigkeit des Logarithmus eines unitären Operators. Nieuw Arch. Wisk., 15, no. 1-2 (1997), 57-61. MR 98j:47036

[6] Ch. Schmoeger: Remarks on commuting exponentials in Banach algebras. Proc Amer. Math. Soc. 127 (1999), 1337-1338. MR 99h:46090

[7] Ch. Schmoeger: Remarks in commuting exponential in Banach algebras, II. Proc. Amer. Math. Soc. 128 (2000), 3405-3409. CMP 2000:17

[8] E. M. E. Wermuth: A remark on commuting operator exponentials. Proc. Amer. Math. Soc. 125 (1997), 1685-1688. MR 97g:39011

Mathematisches Institut I, Universität Karlsruhe, D-76128 Karlsruhe, Germany

E-mail address: christoph.schmoeger@math-uni-karlsruhe.de 